\title{
Performance Dependence on Channel Baud-Rate of Coherent Single-carrier WDM Systems
}

\author{
A. Bononi, N. Rossi, P. Serena \\ Università degli Studi di Parma, Dipartimento di Ingegneria dell'Informazione, via G. Usberti 181/A, \\ 43124 Parma (Italy), $\bowtie$ bononi@tlc.unipr.it
}

\begin{abstract}
The nonlinear threshold versus baudrate is simulated for dual-polarization BPSK, QPSK and 16QAM over $20 \times 100 \mathrm{~km}$ SMF dispersion-managed (DM) and unmanaged (DU) links. The best baudrate is discussed, and comparisons with $D U$ theory are provided.
\end{abstract}

\section{Introduction}

The issue of the best granularity in wavelength division multiplexed (WDM) long-haul coherent optical links, i.e., the per-carrier baud-rate for orthogonal frequency division multiplexing (OFDM) or the per-channel baud-rate for single-carrier modulations has been recently discussed ${ }^{1-5}$. The best baud-rate either minimizes the bit error rate (BER) at a fixed distance, or maximizes the transmission distance at a fixed BER. We recently reported on simulations of the nonlinear threshold (NLT) $\hat{P}_{1}$ (i.e., the transmitted channel power at 1-dB signal to noise ratio (SNR) penalty at $B E R=10^{-3}$ ) versus baud-rate $R$ in polarizationmultiplexed quadrature phase shift keying (PDMQPSK) coherent systems at constant bandwidth efficiency $\eta \triangleq R / \Delta f$ (with $\Delta f$ the channel frequency spacing), with the aim of highlighting the dominant nonlinear effect as the granularity changes ${ }^{6}$. We also showed how the maximum transmission distance in dispersion unmanaged (DU) links can be inferred from the "power budget", i.e., the gap between the NLT at a given transmission distance and the linear threshold at the minimum required SNR in linear propagation ${ }^{7}$. Similar inferences for dispersion managed (DM) systems are well known ${ }^{8}$. Since the linear threshold scales linearly with $R$, the largest power budget (hence the longest reach) is achieved at the baud-rate that maximizes the ratio $\hat{P}_{1} / R$.

In this paper, we extend the NLT simulations of both DM and DU systems in ${ }^{6}$ to two more modulation formats, namely, binary PSK (BPSK) and 16-level quadrature amplitude modulation (16QAM). We present the results in terms of $\hat{P}_{1} / R$ versus $R$ with the aim of highlighting not only the dominant nonlinearity, but also the optimal baudrate. For DU systems, we finally compare our simulations against predictions of the Gaussian Nonlinear (GN) theory ${ }^{9}$.

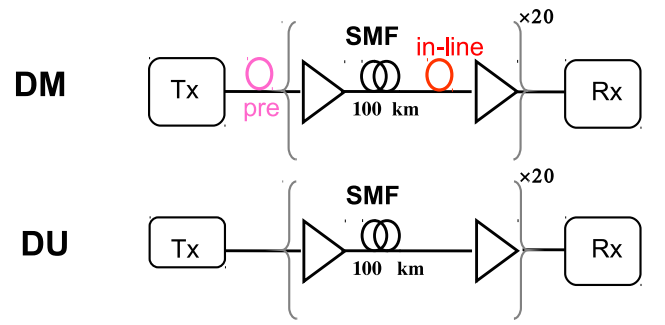

Fig. 1: Block diagrams of DM and DU simulated systems. TX block represents 15 channels, with NRZ pulses modulated at $R$ Gbaud.

\section{Simulated DM and DU systems}

Fig. 1 shows the block diagram of the simulated DM and DU links. The transmitter consisted of $N_{c h}=15$ WDM non-return to zero (NRZ) polarization-multiplexed (PDM) channels at $R$ Gbaud (only at $R=5$ Gbaud we used $N_{c h}=37$ ), with channel spacing $\Delta f=R / \eta$ and bandwidth efficiency $\eta=0.56$. Three modulation formats were considered: BPSK, QPSK and 16QAM. The transmission line consisted of 20 spans of $100 \mathrm{~km}$ of standard single-mode fiber (SMF) $(D=17 \mathrm{ps} / \mathrm{nm} / \mathrm{km})$. In the DM case, an in-line residual dispersion per span (RDPS) of 30 $\mathrm{ps} / \mathrm{nm}$ and optimal precompensation were used. Propagation used the vector split-step Fourier method (SSFM) with zero polarization mode dispersion and Manakov nonlinear step. Other details of the simulated system are the same as in ${ }^{7}$.

For BPSK and QPSK the digital signal processor (DSP) consisted of a chromatic dispersion compensation block, of a data-aided polarization demultiplexer, and a 27-tap Viterbi and Viterbi phase estimator ${ }^{7}$. For 16QAM the DSP had a 15tap data-aided least-squares butterfly equalizer ${ }^{10}$ and a 27 -tap blind-phase search algorithm ${ }^{11}$.

Objective of the simulations was to estimate the NLT $\hat{P}_{1}$ versus baudrate when nonlinearities $(\mathrm{NL})$ are selectively activated ${ }^{12}$. The NLT is obtained from a series of BER Monte Carlo estimations (averaged over input polarization states) 
at increasing amplifiers noise figure until the target BER is obtained. Using nonlinearity decoupling, we studied the following four cases: 1) single channel (label "SPM"); 2) WDM with only scalar XPM (label "XPM"); 3) WDM with only cross-polarization modulation (label "XPolM"); 4) WDM with all nonlinearities (label "WDM"). Simulations were run using the open-source software Optilux ${ }^{13}$

\section{Results}

DM system. The top row of Fig. 2 shows the normalized NLT $\hat{P}_{1} / R$ versus baud-rate $R$ in a legacy DM map. In the figure we report both the unrealistic case of noise loading (solid lines) and the realistic case of amplified spontaneous emission (ASE) noise distributed at each amplifier, where nonlinear signal-ASE interactions are fully accounted for (dashed lines). The figure shows that:

i) scalar XPM plays a minor role in the BPSK, QPSK constant-intensity formats, and is quite sensitive to signal-noise interactions, here manifested as nonlinear phase noise (NLPN) ${ }^{6}$. It becomes the dominant NL effect at lower baud-rates in 16QAM (the extra noise-induced intensity fluctuations become a second-order effect, hence no
NLPN dependence is observed);

ii) single channel effects (SPM) dominate above 28Gbaud with 16QAM, above 40Gbaud with QPSK and above 100Gbaud with BPSK. They are quite sensitive to NLPN only with PSK formats and at lower rates where they are negligible: $\equiv$

iiii) for PSK formats the dominant nonlinearity is XPolM (which is not impacted by signal-ASE interactions) at lower $R$, and SPM at larger $R$ (where its sensitivity to NLPN disappears). For 16QAM scalar XPM is slightly larger but comparable to XPolM up to 28Gbaud;

iv) with all three WDM homogeneous formats the NLPN dependence is negligible, and $\hat{P}_{1} / R$ is roughly constant (within $1 \mathrm{~dB}$ ) over the entire baud-rate range, with a very shallow maximum around 28 Gbaud.

DU system. Fig. 2 (bottom row) shows the corresponding $\hat{P}_{1} / R$ versus $R$ curves. Here ASE was loaded at the receiver since signal-noise nonlinear interactions are negligible ${ }^{12}$. The figure shows that:

i) with all formats, scalar XPM tends to dominate over XPoIM, although both effects are of comparable size;

ii) single-channel nonlinearity is comparable
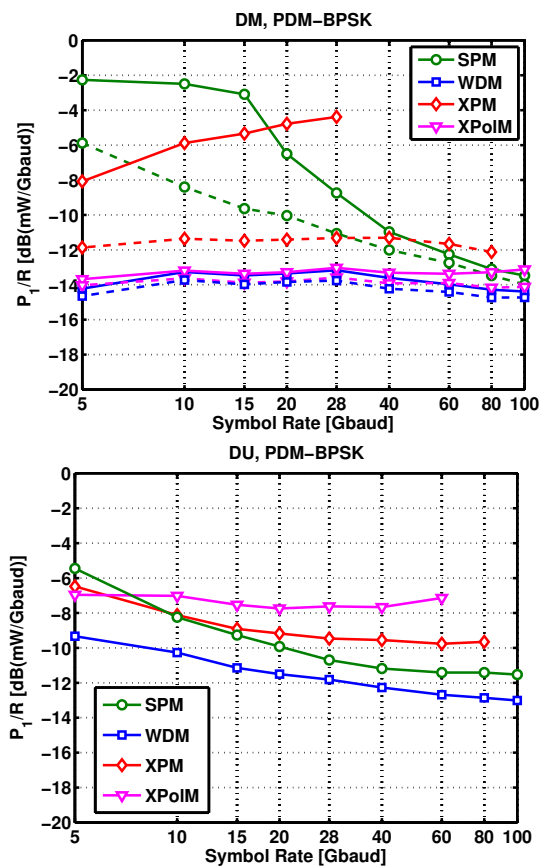
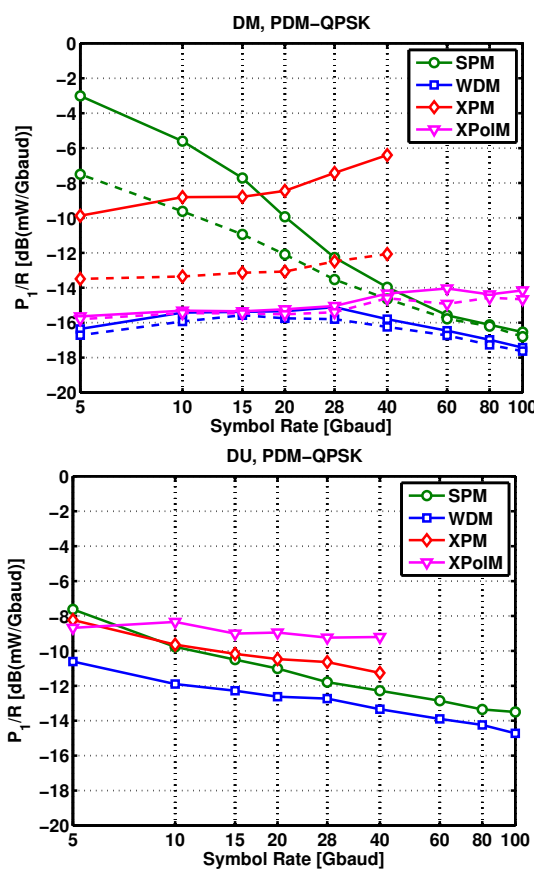
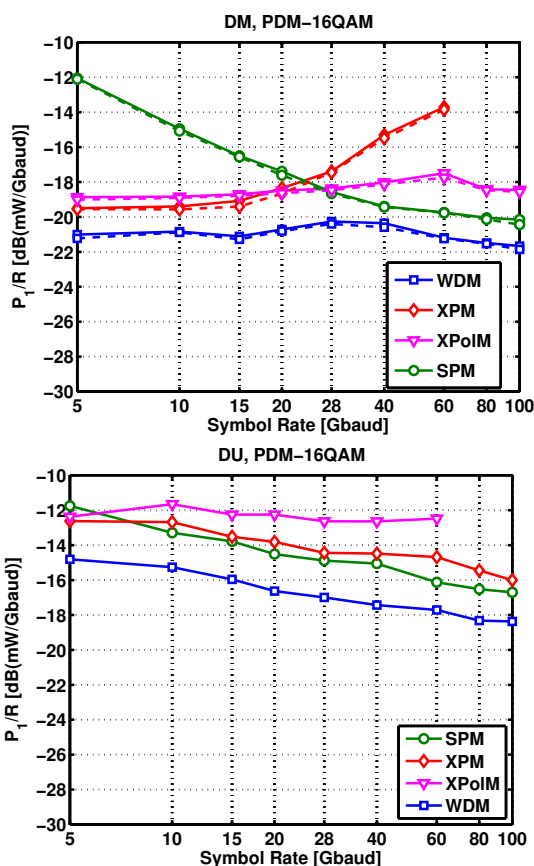

Fig. 2: $1 \mathrm{~dB}-\mathrm{NLT} \hat{P}_{1}$ divided by baudrate $R$ vs baudrate for central channel of 15-channel homogeneous NRZ system with uniform channel spacing $\Delta f=R / \eta$, and bandwidth fill factor $\eta=0.56$, over a 20x100km SMF link. Top row: DM with $30 \mathrm{ps} / \mathrm{nm}$ RDPS and distance-optimized precompensation; Bottom row: DU. Left: PDM-BPSK. Center: PDM-QPSK; Right: PDM-16QAM. Solid lines: ASE noise loading at RX. Dashed lines: distributed ASE. 
to cross-channel nonlinearities (since XPM and XPolM are correlated, their joint NLT is not a simple linear composition of the individual NLTs);

iv) with all formats the $\hat{P}_{1} / R$ WDM curves (all $\mathrm{NL}$ effects) are decreasing with $R$, i.e., the best baud-rate is below $5 \mathrm{Gbaud}$.

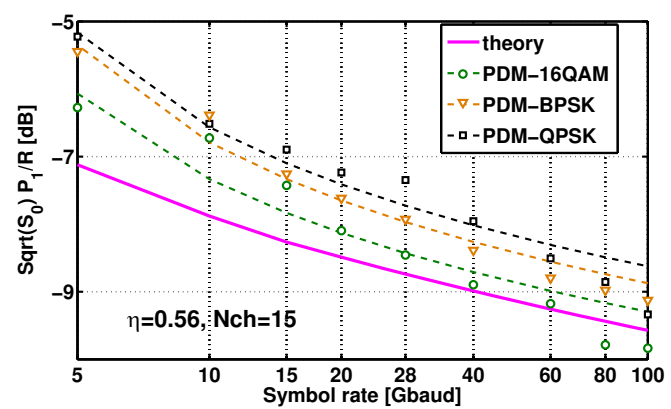

Fig. 3: Plot of $\sqrt{S_{0}} \hat{P}_{1} / R$ [dB(mW/Gbaud)] versus $R$ [Gbaud] for DU $20 \times 100 \mathrm{~km}$ SMF line. Simulations at $\eta=0.56$ and $N_{c h}=15$. Theory plots $1 / \sqrt{3 c^{2} a_{N L}}$, with $a_{N L}$ analytically calculated ${ }^{9}$.

For DU systems, a GN theory ${ }^{9}$ is available to cross-verify the simulation results. According to theory, $\hat{P}_{1}=1 / \sqrt{3 c^{2} S_{0} a_{N L}}$ ( ${ }^{7}$, eq. (7),(9)) where $c=1.27, S_{0}$ is the Hinear electrical SNR $\equiv$ ieving the target BER, and $a_{N L}$ is the nonlinear interference coefficient. If $a_{N L}$ were format-independent, the quantity $\sqrt{S_{0}} \hat{P}_{1}$ would also be format-independent. Taking data from Fig. 2 and using the actual simulation values $S_{0}=[7.77,10.77,17.07] \mathrm{dB}$ for BPSK, QPSK and 16QAM, respectively, Fig. 3 plots $\sqrt{S_{0}} \hat{P}_{1} / R$ versus $R$. The theoretical solid-line curve plots $1 / \sqrt{3 c^{2} a_{N L} R^{2}}$ with $a_{N L}=\int G_{N L I}(f) d f$ calculated as in $\left({ }^{9}\right.$, eq. (1)) with the same normalized WDM power spectral density $G_{W D M}(f)$ used for the simulations in Fig. 2. Since approximately ${ }^{9} a_{N L} R^{2} \propto \log \left(k_{1} R^{2}\right)$, dashed lines provide a least-squares interpolation of the simulated data according to $k_{2} / \sqrt{\log \left[k_{1} R^{2}\right]}$. From the figure we learn that the theoretical monotone decreasing trend versus $R$ in DU systems at $\eta=0.56$ is confirmed with all three modulation formats. Theory assumes a Gaussian input process, so not surprisingly the 16QAM format gets closer to the theoretical curve than the PSK formats. The figure also indirectly confirms that the NLI coefficient $a_{N L}$ is indeed format-dependent, with a uniform spread of about $1 \mathrm{~dB}$ over the shown baud-rate range and formats.

Part of the reason why NLT increases (i.e., NL weakens) at lower baud-rates in DU simulations is that the number of channels is fixed, hence the total occupied bandwidth at constant $\eta$ shrinks with

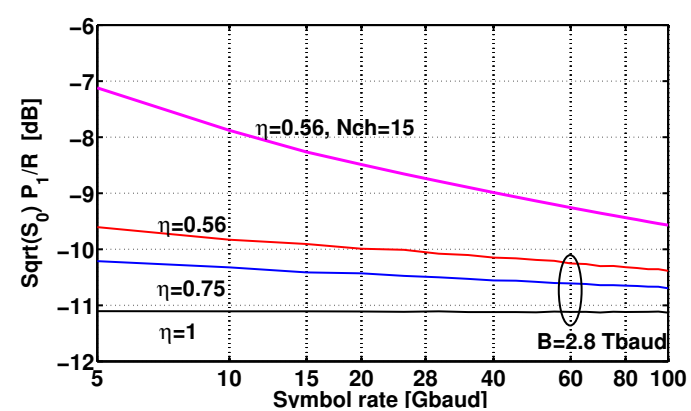

Fig. 4: Plot of $\sqrt{S_{0}} \hat{P}_{1} / R$ versus $R\left(\mathrm{GN}\right.$ theory ${ }^{9}$ ) for DU $20 \times 100 \mathrm{~km}$ SMF line at varying bandwidth efficiency $\eta$ and fixed aggregate baud-rate $B=N_{c h} R=2.8$ Tbaud. Magenta curve same as in Fig. 3.

R. However, the GN theoretical curves in Fig. 4 (where the magenta curve is the same as in Fig. 3 ) show that even when we keep a fixed WDM aggregate baudrate $B=N_{c h} R$ and then increase $N_{c h}$ when $R$ is reduced, still the $\sqrt{S_{0}} \hat{P}_{1} / R$ plot versus $R$ remains monotone-decreasing at any $\eta<1$, with a limiting flat behavior in the NyquistWDM case $\eta=1$. This implies that the tilt of $\hat{P}_{1} / R$ decreases at increasing bandwidth efficiency $\eta$, i.e., the baud-rate dependence of maximum reach becomes less evident.

\section{Conclusions}

We studied by simulation the dominant nonlinearity in homogeneous BPSK, QPSK and 16QAM WDM DM and DU systems at a bandwidth efficiency $\eta=0.56$. We found that the NLT is not sensitive to nonlinear signal-noise interactions. Plots of $N L T / R$ versus per-channel baud-rate $R$ lead us to infer that system reach is very weakly dependent on $R$ in practical DM and DU SMF coherent systems.

\section{References}

1 L. B. Du et al., Opt. Exp. 19, 8079 (2011).

2 A. D. Ellis et al., Proc. OECC'10 462-463 (2010)

3 S. L. Jansen, Proc. OFC'12 OTh1B.1 (2012)

4 Y. Tang et al., Photon. Technol. Lett. 22, 1250 (2010).

5 P. Poggiolini et al., Photon. Technol. Lett. 23, 15 (2011).

6 A. Bononi et al.,Proc. OFC'11 OW07 (2011).

7 A. Bononi et al., Opt. Exp. 20, B204 (2012).

8 J.-C. Antona et al., Comptes Rendus 9, 963 (2008).

9 P. Poggiolini, J. Lightw. Technol. 30, 3857 (2012).

10 C. R. Johnson et al., Software Receiver Design, www.scribd.com/doc/54305167/SoftwareDefined-Radio, p. 181.

11 T. Pfau et al., J. Lightw. Technol. 27, 989 (2009).

12 A. Bononi et al.,Proc. ECOC'10Th.10.E.1 (2010).

13 "Optilux Toolbox", www.optilux.sourceforge.net 